\section{(C) OPEN ACCESS}

\title{
Midlife blood pressure predicts future diastolic dysfunction independently of blood pressure
}

\author{
Arjun Kumar Ghosh, ${ }^{1,2}$ Alun David Hughes, ${ }^{3}$ Darrel Francis, ${ }_{1}^{1}$ Nishi Chaturvedi, ${ }^{3}$ \\ Denis Pellerin, ${ }^{2}$ John Deanfield, ${ }^{3}$ Diana Kuh, ${ }^{4}$ Jamil Mayet, ${ }^{1}$ Rebecca Hardy, ${ }^{4}$ \\ on behalf of the MRC NSHD Scientific and Data Collection Team
}

- Additional material is published online only. To view please visit the journal online (http://dx.doi.org/10.1136/ heartjnl-2015-308836).

${ }^{1}$ International Centre for Circulatory Health, National Heart and Lung Institute, Imperial College, London, UK ${ }^{2}$ Barts Heart Centre, St Bartholomew's Hospital, Barts Health NHS Trust, London, UK ${ }^{3}$ Institute of Cardiovascular Science, University College London, London, UK ${ }^{4}$ Medical Research Council Unit for Lifelong Health and Ageing, University College London, London, UK

\section{Correspondence to} Dr Arjun Kumar Ghosh, Department of Cardiology, Barts Heart Centre, St Bartholomew's Hospital, West Smithfield, London EC1A 7BE, UK; a.ghosh@imperial.ac.uk

Received 17 October 2015 Revised 2 March 2016 Accepted 14 March 2016 Published Online First 7 April 2016
CrossMark

To cite: Ghosh AK, Hughes AD, Francis $D$, et al. Heart 2016;102:

$1380-1387$.

\begin{abstract}
Objectives High blood pressure (BP) is associated with diastolic dysfunction, but the consequence of elevated BP over the adult life course on diastolic function is unknown. We hypothesised that high BP in earlier adulthood would be associated with impaired diastolic function independent of current BP.

Methods Participants in the Medical Research Council National Survey of Health and Development birth cohort $(n=1653)$ underwent investigations including

echocardiography at age 60-64 years. The relationships between adult BP, antihypertensive treatment (HTT) and echocardiographic measures of diastolic function were assessed using adjusted regression models.
\end{abstract}

Results Increased systolic BP (SBP) at ages 36, 43 and 53 years was predictive of increased $\mathrm{E} / \mathrm{e}^{\prime}$ and increased left atrial volume. These effects were only partially explained by SBP at 60-64 years and increased left ventricular mass. HTT was also associated with poorer diastolic function after adjustment for SBP at 60-64 years. Faster rates of increase in SBP in midlife were also associated with increased poorer diastolic function.

Conclusions High SBP in midlife is associated with poorer diastolic function at age 60-64 years. Early identification of individuals with high BP or rapid rises in BP may be important for prevention of impaired cardiac function in later life.

\section{INTRODUCTION}

With an ageing population, and better postmyocardial infarction survival, the burden of congestive heart failure is increasing and represents a major public health challenge. ${ }^{1}$ Diastolic dysfunction is common in people over 45 years with a prevalence of $28 \%$ in a large community study, ${ }^{1}$ and is a precursor of heart failure with preserved ejection fraction (HFPEF). ${ }^{2}$ Echocardiographic measures of diastolic dysfunction and elevated left ventricular (LV) filling pressure also predict a threefold increased cardiac mortality and a twofold increased all-cause mortality in those with normal ejection fractions. ${ }^{2}{ }^{3}$ High systolic blood pressure (SBP) is an important cause of diastolic dysfunction, and there is evidence that raised antecedent SBP in early and midadulthood is associated with increased risk of heart failure, ${ }^{4}$ and elevated risk of cardiovascular mortality independent of current blood pressure (BP). ${ }^{5}$ However, there has been limited work investigating the effects of longitudinal changes in
BP over adulthood on diastolic function. ${ }^{6}$ We have previously shown that increased SBP in adult midlife is associated with increased LV mass in later life independent of later SBP and that rapid rises in midlife SBP might play a key role. ${ }^{7}$ We hypothesised that a similar relationships may exist between SBP and diastolic function and also aimed to determine the extent to which this might be due to increased LV mass. In addition, we examined the association between antihypertensive treatment (HTT) and subsequent diastolic function.

\section{METHODS}

Study patients

The UK Medical Research Council National Survey of Health and Development (MRC NSHD) is a prospective birth cohort study of singleton births that occurred in 1 week of March 1946 in England, Scotland and Wales (5362 births; 2547 women, 2815 men). Follow-up has included over 20 contacts with the whole cohort between birth and the most recent data collection when the participants were between 60 and 64 years of age.

Study members still alive and with a known current address in England, Scotland or Wales were invited for an assessment at one of six clinical research facilities (CRFs) or to be visited by a research nurse at home. Invitations were not sent to those who had died $(\mathrm{N}=778)$, who were living abroad $(\mathrm{N}=570)$, had previously withdrawn from the study $(\mathrm{N}=594)$ or had been lost to follow-up $(\mathrm{N}=564)$. Of the 2856 invited participants, 2229 (78\%) were assessed: 1690 (59\%) attended a CRF and the remaining 539 were visited at home. Echocardiography was only carried out at the CRF $(\mathrm{N}=1653)$. The participating sample remains broadly representative of native born British men and women of the same age. ${ }^{8}$

Ethical approval was obtained from the Central Manchester Research Ethics Committee (07/ H1008/168) and the Scotland A Research Ethics Committee. Written informed consent was obtained from each study member at each stage of data collection.

\section{Anthropometry and BP measurement}

Height and weight were measured at the clinic visit and body mass index (BMI) calculated. Sitting brachial BP was measured in the upper right arm with an appropriately sized cuff after $5 \mathrm{~min}$ of rest at 53 years and 60-64 years with the second 
measurement used in analyses, or the first measure where the second was missing. A Hawksley random zero sphygmomanometer (Hawksley \& Sons, Lancin, UK; a state of the art machine at the time) was used at 36 and 43 years. ${ }^{5} 9$ A validated oscillometric device (Omron HEM-705) was used in later in home visits. To enable comparison of BP measured by the different machines, the measurements from the random zero sphygmomanometer were adjusted using published conversion equations. $^{10}$

\section{Antihypertensive treatment and diabetes status}

Prior to clinic attendance, study members completed a postal questionnaire that included details of current medication. Antihypertensive medications for the last two rounds were classified according to International Classification of Diseases and related Health Problems classification. ${ }^{11}$ Self-reported and doctor diagnosed type 2 diabetes mellitus (T2DM) was obtained from the postal questionnaires and reports at earlier follow-ups.

\section{Echocardiography}

Of the 1690 participants who attended a clinic, 1653 (798 men and 855 women, mean age $63.3 \pm 1.1$ years (1SD)) underwent echocardiography using GE Vivid I machines (GE, Connecticut, USA) and $1576 \mathrm{had}$ at least one analysable image (95\%). Echocardiographic images were obtained from parasternal long axis and short axis, apical five-chamber, four-chamber, threechamber, two-chamber and aortic views along with conventional and tissue Doppler in the four-chamber view. Image analysis was carried out by three experienced British Society of Echocardiography-accredited readers including the author (AKG) masked to patient identity using GE EchoPac software.

The following markers of diastolic function were measured in accordance with American Society of Echocardiography/ European Association of Echocardiography recommendationsratio of early (E) to late (A) transmitral Doppler flow (E/A), early $\left(\mathrm{e}^{\prime}\right)$ myocardial velocity at the mitral valve annulus (average of septal and lateral wall measures) $\mathrm{e}^{\prime}, \mathrm{E} / \mathrm{e}^{\prime}$ and ratio of early and late $\left(a^{\prime}\right)$ myocardial velocities at the mitral valve annulus (average of septal and lateral wall measures) $\mathrm{e}^{\prime} / \mathrm{a}^{\prime} .{ }^{12} \mathrm{E} / \mathrm{e}^{\prime}$ was calculated as an estimate of LV filling pressure. ${ }^{12}$ Left atrial volume indexed to body surface area (LAVI) was also examined as a marker of chronically elevated LV filling pressures. ${ }^{13}$

Quality control measures included standardised training for senior, experienced echocardiographers and readers, echocardiographer observation by trained echocardiographers, periodic reader and echocardiographer review and refresher sessions, phantom studies on ultrasound machines and continuous quality control audit throughout the period of data collection. Blind duplicate reading reproducibility studies ( $n=70$ on two occasions) were carried out to establish inter-reader and intrareader variability. These showed excellent reproducibility (intraclass correlation coefficients were $>0.90$ for most measurements).

\section{Statistical analysis}

Statistical analysis was performed using Stata V.14.1 (StataCorp LP, USA). Initially, separate regression models investigated the association between SBP at each of the 4 ages at which it was measured and measures of diastolic function at 60-64 years, with adjustment for sex, age at clinic visit and clinic attended. We examined whether associations were linear by inspection of residuals. Given evidence that cardiac function may differ by sex, ${ }^{1}$ we also investigated whether associations with SBP were subject to effect modification by sex through inclusion of a sex $\times$ SBP term in models, but this was not statistically significant in any model. Consequently, we show data from models including both sexes that were adjusted for sex. HTT at the same age as BP measurement was then added to the models. Additional models assessed whether earlier SBP remained predictive once current SBP was also included, that is, to what extent antecedent SBP was independent of current SBP. This is an important consideration given the anticipated correlations between SBP at different times within an individual (tracking). Further statistical models were constructed by including potential confounders BMI, T2DM, smoking and physical activity status at age 60-64 years. In a further model left ventricular mass indexed to body surface area (LVMI) was included with possible confounders to explore the extent to which LVMI might mediate the associations observed.

In order to maintain the sample size and minimise bias introduced by missing data in fully adjusted analyses, we employed a multiple imputation procedure to impute missing covariates. For each outcome, a total of 20 imputed datasets were obtained using chained equations implemented using imputation by chained equations in Stata. For the imputation models we included all variables in the final adjusted analytic model, each model, as well as the outcome and additional variables that helped predict the missing covariates. The regression coefficients and standard errors were calculated for each imputed dataset, and then combined using Rubin's rule.

To investigate whether rate of change in SBP at a particular period of midlife was more strongly associated with diastolic function, we calculated the change in SBP for the periods 36-43 years, 43-53 years and 53-60/64 years conditional on earlier SBP by modelling each SBP measure (from age 43 years) on the earlier measure(s) for each sex and saving the residuals. These residuals reflect SBP velocity and can be interpreted as the change in SBP in an individual above or below that expected on average in the sample given their earlier SBP. ${ }^{14}$ The residuals were standardised (mean $=0$ and $\mathrm{SD}=1$ ) to allow a comparison of the relative strength of associations between periods. We subsequently fitted regression models including all these standardised changes with each of the measures of diastolic function as the outcome. This analysis was performed only for individuals who had all variables observed (complete case analysis). Two models were constructed: model 1-adjusted for age, sex and CRF attended; model 2-model 1+T2DM+BMI+smoking status + physical activity status+current HTT. p Values were calculated using Wald tests. Similar analyses were repeated for diastolic BP (DBP), pulse pressure (PP) and mean arterial pressure (MAP).

Sensitivity analyses were carried out to assess whether the associations with SBP remained unchanged if those who were hypertensive $(\mathrm{SBP} \geq 140 \mathrm{~mm} \mathrm{Hg}$ or $\mathrm{DBP} \geq 90 \mathrm{~mm} \mathrm{Hg})$ were excluded.

\section{RESULTS}

Characteristics of participants with any echocardiography data at age 60-64 years are shown in table 1 . Those with unanalysable echocardiograms had higher BMIs and heart rates, but BP did not differ. ${ }^{7}$ Additional participant characteristics are presented in online supplementary table S1.

SBP from age 53 years was negatively associated with $\mathrm{e}^{\prime}$ (table 2). This relationship weakened with progressive risk factor adjustment. HTT was negatively associated with $\mathrm{e}^{\prime}$ in the minimally adjusted model from age 53 years.

Broadly similar findings were observed when the associations between current and antecedent SBP and E/A were examined, although relationships tended to be weaker. SBP at 53 and 
Table 1 Echocardiographic and cardiac risk factor characteristics of study participants

\begin{tabular}{|c|c|c|c|c|c|c|}
\hline \multirow[b]{2}{*}{ Variable (at age $60-64$ years unless stated otherwise) } & \multicolumn{2}{|l|}{ All } & \multicolumn{2}{|l|}{ Men } & \multicolumn{2}{|c|}{ Women } \\
\hline & $\mathbf{n}$ & Result & $\mathbf{n}$ & Result & $\mathbf{n}$ & Result \\
\hline Age, years & 1626 & $63.2(1.1)$ & 786 & $63.2(1.2)$ & 840 & $63.3(1.1)$ \\
\hline $\mathrm{BMI}, \mathrm{kg} / \mathrm{m}^{2}$ & 1633 & $27.7(4.6)$ & 791 & $27.7(4.0)$ & 842 & $27.6(5.2)$ \\
\hline SBP, mm Hg & 1633 & $135.7(18.0)$ & 791 & $139.0(17.8)$ & 842 & $132.7(17.6)$ \\
\hline SBP in those on HTT, $\mathrm{mm} \mathrm{Hg}$ & 347 & $137.4(17.4)$ & 179 & $138.2(16.2)$ & 168 & $131.8(16.9)$ \\
\hline SBP in those not on HTT, mm Hg & 1120 & $134.9(18.0)$ & 518 & $136.1(17.6)$ & 602 & $129.9(15.8)$ \\
\hline SBP in those with unknown HTT status, $\mathrm{mm} \mathrm{Hg}$ & 166 & $137.4(18.6)$ & 92 & $138.1(17.3)$ & 74 & $132.1(17.2)$ \\
\hline SBP at age 53 years, $\mathrm{mm} \mathrm{Hg}$ & 1537 & $134.3(19.1)$ & 738 & $138.0(18.9)$ & 799 & $130.8(18.5)$ \\
\hline SBP at age 43 years, $\mathrm{mm} \mathrm{Hg}$ & 1522 & $123.7(14.1)$ & 735 & $128.1(13.1)$ & 787 & $119.6(13.6)$ \\
\hline SBP at age 36 years, $\mathrm{mm} \mathrm{Hg}$ & 1479 & $120.0(13.7)$ & 714 & $125.8(12.8)$ & 765 & $114.6(12.3)$ \\
\hline $\mathrm{DBP}, \mathrm{mm} \mathrm{Hg}$ & 1633 & $77.3(9.7)$ & 791 & $79.0(9.8)$ & 842 & $75.7(9.3)$ \\
\hline $\mathrm{DBP}$ at age 53 years, $\mathrm{mm} \mathrm{Hg}$ & 1537 & $83.5(11.9)$ & 738 & $86.4(11.9)$ & 799 & $80.9(11.2)$ \\
\hline DBP at age 43 years, $\mathrm{mm} \mathrm{Hg}$ & 1522 & $80.5(9.5)$ & 735 & $83.4(9.0)$ & 787 & $77.8(9.1)$ \\
\hline DBP at age 36 years, $\mathrm{mm} \mathrm{Hg}$ & 1477 & $78.3(9.6)$ & 713 & $80.8(9.4)$ & 764 & $75.9(9.3)$ \\
\hline Heart rate, bpm & 1630 & $68.9(11.2)$ & 789 & $67.4(11.3)$ & 841 & $70.3(10.9)$ \\
\hline IVSD, cm & 1473 & $1.1(0.2)$ & 701 & $1.1(0.2)$ & 772 & $1.0(0.2)$ \\
\hline PWT, cm & 1471 & $1.0(0.2)$ & 699 & $1.1(0.2)$ & 772 & $0.9(0.2)$ \\
\hline RWT & 1469 & $0.4(0.1)$ & 699 & $0.4(0.1)$ & 770 & $0.4(0.1)$ \\
\hline LVM, g & 1471 & $181.3(59.3)$ & 699 & $209.3(60.3)$ & 772 & $156.0(45.4)$ \\
\hline LVMI, g/m² & 1471 & $95.7(26.6)$ & 699 & $104.1(27.9)$ & 772 & $88.1(22.8)$ \\
\hline Ejection fraction (\%) & 1459 & $68.7(9.7)$ & 692 & $67.2(10.1)$ & 767 & $69.9(9.2)$ \\
\hline E/A & 1576 & $1.0(0.3)$ & 755 & $1.0(0.3)$ & 821 & $1.0(0.3)$ \\
\hline $\mathrm{e}^{\prime}, \mathrm{cm} / \mathrm{s}$ & 1533 & $8.8(1.9)$ & 727 & $8.9(1.9)$ & 806 & $8.8(1.9)$ \\
\hline $\mathrm{E} / \mathrm{e}^{\prime}$ & 1490 & $7.9(2.1)$ & 701 & $7.5(2.0)$ & 789 & $8.3(2.1)$ \\
\hline $\mathrm{e}^{\prime} / \mathrm{a}^{\prime}$ & 1507 & $0.8(0.2)$ & 709 & $0.8(0.2)$ & 798 & $0.9(0.2)$ \\
\hline $\mathrm{LAVI}, \mathrm{mL} / \mathrm{m}^{2}$ & 1417 & $21.1(7.2)$ & 701 & $22.0(7.4)$ & 716 & $20.2(7.0)$ \\
\hline
\end{tabular}

60-64 years was predictive of E/A and HTT at any age was unrelated to $\mathrm{E} / \mathrm{A}$ (table 3). Data relating SBP measured at various ages to $\mathrm{e}^{\prime} / \mathrm{a}^{\prime}$ were consistent with observations for $\mathrm{e}^{\prime}$ and E/A (data not shown).

SBP from 36 years onwards was positively associated with increased $\mathrm{E} / \mathrm{e}^{\prime}$ (table 4). The relationship persisted after adjustment for age, sex, clinic attended, current HTT/SBP/BMI/ T2DM/smoking/physical activity. SBP at age 53 years and HTT at age 53 years were also positively associated with LAVI (table 5). Analyses were repeated replacing SBP with DBP, PP or MAP should similar associations (data not shown).

From 43 years onwards, those on HTT had increased E/ $\mathrm{e}^{\prime}$; this was not affected substantively by adjustment for current SBP; regression coefficients were slightly attenuated by adjustment for BMI/T2DM/smoking/physical activity although relationships remained statistically significant (table 4). We also investigated whether the effect of earlier SBP was mediated via LV hypertrophy by inclusion of LVMI into regression models. This was not the case.

We investigated whether relationships between elevated BP in earlier life and $\mathrm{e}^{\prime}$ and $\mathrm{E} / \mathrm{e}^{\prime}$ might be driven by inclusion of people who went on to develop hypertension. However, when those who were hypertensive at 60-64 years (SBP $\geq 140 \mathrm{~mm} \mathrm{Hg}$ or DBP $\geq 90 \mathrm{~mm} \mathrm{Hg}$ ) were excluded from the analysis, the associations remained very similar (data not shown).

We looked to see whether there was a sensitive period when rate of change in SBP had most influence on diastolic function and filling pressure. Increased rates of rise in SBP over the age periods 43-53 and 53-60/64 years were also significantly associated with worse $\mathrm{e}^{\prime}$ (figure 1A) and E/A (figure 1B). Increased rate of rise in SBP at all age periods (36-43 years, 43-53 years and 53-60/64 years) was associated with increased $\mathrm{E} / \mathrm{e}^{\prime}$ (figure 1C), although the relationship was strongest over the period $43-53$ years in the minimally adjusted model. The rate of rise in the 43-53 years period was also associated with increased LAVI (figure 1D).

\section{DISCUSSION}

BP from early midlife predicted worse diastolic function and evidence of elevated filling pressure at age 60-64 years. This effect was independent of current BP, extended across the whole range of BP, and was not limited to people with hypertension or those who went on to develop hypertension at the time when diastolic function was assessed. We observed that those on HTT from early midlife had poorer diastolic function than those not on HTT even after current BP was accounted for. Further, we found that individuals showing comparatively rapid rises in SBP between 43-53 years and 53-60/64 years had worse subsequent diastolic function irrespective of their absolute level of SBP. Since the rise in BP in the UK population typically begins to accelerate around the fourth decade of life until it slows again in later life, ${ }^{15}$ we suggest that the period 40-60 years may represent a sensitive period when an accelerated rise in BP adversely influences future development of diastolic dysfunction.

There has been limited work in the past on relating antecedent BP with future diastolic function. Arnlöv et al reported that higher SBP and DBP at the age of 50 years were associated 
Table 2 Regression between $\mathrm{e}^{\prime}$ at 60-64 years and SBP and antihypertensive treatment at four time points with further adjustment for covariables

\begin{tabular}{|c|c|c|c|c|c|c|c|c|c|c|c|}
\hline \multirow{2}{*}{\multicolumn{2}{|c|}{ Independent variable }} & \multicolumn{2}{|l|}{ Model 1} & \multicolumn{2}{|l|}{ Model 2} & \multicolumn{2}{|l|}{ Model 3} & \multicolumn{2}{|l|}{ Model 4} & \multicolumn{2}{|l|}{ Model 5} \\
\hline & & $\boldsymbol{\beta}(95 \% \mathrm{Cl})$ & $\mathrm{p}$ Value & 及 $(95 \% \mathrm{Cl})$ & $\mathrm{p}$ Value & $\beta(95 \% \mathrm{Cl})$ & $\mathrm{p}$ Value & 及 $(95 \% \mathrm{Cl})$ & $\mathrm{p}$ Value & 及 $(95 \% \mathrm{Cl})$ & $\mathrm{p}$ Value \\
\hline Age $36(n=1533)$ & SBP & $-0.006(-0.014$ to 0.002$)$ & 0.125 & $-0.006(-0.014$ to 0.002$)$ & 0.122 & $-0.001(-0.009$ to 0.007$)$ & 0.855 & $-0.002(-0.010$ to 0.006$)$ & 0.619 & $0.000(-0.008$ to 0.008$)$ & 0.931 \\
\hline Age $43(n=1533)$ & SBP & $-0.006(-0.013$ to 0.002$)$ & 0.128 & $-0.005(-0.012$ to 0.002$)$ & 0.171 & $0.002(-0.067$ to 0.010$)$ & 0.598 & 0.001 (-0.007 to 0.008$)$ & 0.816 & $0.000(-0.009$ to 0.008$)$ & 0.959 \\
\hline Age 53 (n=1533) & SBP & $-0.011(-0.017$ to -0.006$)$ & $<0.001$ & $-0.010(-0.016$ to -0.005$)$ & $<0.001$ & $-0.005(-0.011$ to 0.001$)$ & 0.084 & $-0.004(-0.010$ to 0.002$)$ & 0.157 & -0.002 ( -0.006 to 0.009$)$ & 0.694 \\
\hline Age 60-64 ( $n=1533)$ & SBP & $-0.017(-0.022$ to -0.012$)$ & $<0.001$ & $-0.017(-0.022$ to -0.011$)$ & $<0.001$ & NR & NR & $-0.015(-0.020$ to -0.009$)$ & $<0.001$ & $-0.014(-0.019$ to -0.008$)$ & $<0.001$ \\
\hline Age 36 & HTT & NR & NR & $0.112(-0.846$ to 1.070$)$ & 0.819 & 0.018 (-0.937 to 0.973$)$ & 0.971 & 0.039 ( -0.910 to 0.989$)$ & 0.935 & $-0.110(-1.157$ to 0.937$)$ & 0.837 \\
\hline Age 43 & HTT & NR & NR & $-0.619(-1.296$ to 0.058$)$ & 0.073 & $-0.598(-1.271$ to 0.075$)$ & 0.081 & $-0.495(-1.163$ to 0.173$)$ & 0.146 & $-0.403(-1.064$ to 0.257$)$ & 0.231 \\
\hline Age 53 & HTT & NR & NR & $-0.333(-0.635$ to -0.032$)$ & 0.030 & $-0.331(-0.630$ to -0.032$)$ & 0.030 & $-0.296(-0.598$ to 0.006$)$ & 0.054 & $-0.230(-0.533$ to 0.073$)$ & 0.136 \\
\hline Age 60-64 & HTT & NR & NR & $-0.253(-0.490$ to -0.016$)$ & 0.036 & NR & NR & $-0.162(-0.407$ to 0.082$)$ & 0.192 & $-0.094(-0.340$ to 0.152$)$ & 0.453 \\
\hline
\end{tabular}

NR

$0.162(-0.407$ to 0.082$)$

$0.192-0.094(-0.340$ to 0.152$)$

model 1

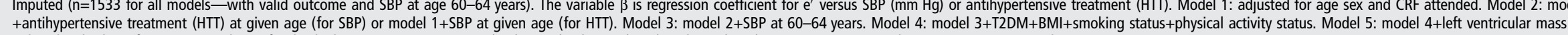
indexed to body surface area. Numbers of individuals receiving HTT were $51(2 \%), 107(3 \%), 438(15 \%)$ and $640(27 \%)$ at age $36,43,53$ and $60-64$ years, respectively.

BMI, body mass index; CRF, clinical research facility; NR, not relevant; SBP, systolic blood pressure; T2DM, type 2 diabetes mellitus.

Table 3 Regression between E/A at 60-64 years and SBP and antihypertensive treatment at four time points with further adjustment for covariables

\begin{tabular}{|c|c|c|c|c|c|c|c|c|c|c|c|}
\hline \multirow[b]{2}{*}{ Independent variable } & & \multicolumn{2}{|l|}{ Model 1} & \multicolumn{2}{|l|}{ Model 2} & \multicolumn{2}{|l|}{ Model 3} & \multicolumn{2}{|l|}{ Model 4} & \multicolumn{2}{|l|}{ Model 5} \\
\hline & & $\boldsymbol{\beta}(95 \% \mathrm{Cl}) \times 10^{-3}$ & $p$ Value & $\boldsymbol{\beta}(95 \% \mathrm{Cl}) \times 10^{-3}$ & $p$ Value & $\boldsymbol{\beta}(95 \% \mathrm{Cl}) \times 10^{-3}$ & $\mathrm{p}$ Value & $\boldsymbol{\beta}(95 \% \mathrm{Cl}) \times 10^{-3}$ & p Value & $\boldsymbol{\beta}(95 \% \mathrm{Cl}) \times 10^{-3}$ & p Value \\
\hline Age $36(n=1576)$ & SBP & 0.3 ( -0.8 to 1.5$)$ & 0.564 & $0.3(-0.8$ to 1.4$)$ & 0.598 & $0.9(-0.2$ to 2.1$)$ & 0.116 & 0.8 (-0.4 to 1.9$)$ & 0.190 & $0.8(-0.3$ to 2.0$)$ & 0.170 \\
\hline Age $43(n=1576)$ & SBP & $0.4(-0.7$ to 1.4$)$ & 0.481 & $0.4(-0.6$ to 1.5$)$ & 0.443 & $1.3(0.2$ to 2.3$)$ & 0.024 & 1.1 (0.06 to 2.2$)$ & 0.039 & $1.2(0.1$ to 2.2$)$ & 0.036 \\
\hline Age $53(n=1576)$ & SBP & $-1.4(-2.2$ to -0.7$)$ & $<0.001$ & $-1.5(-2.3$ to -0.7$)$ & $<0.001$ & $-1.0(-1.8$ to -0.1$)$ & 0.025 & $-0.7(-1.5$ to 0.1$)$ & 0.099 & $-0.7(-1.5$ to 0.2$)$ & 0.118 \\
\hline Age $60-64(n=1576)$ & SBP & $-1.8(-2.6$ to -1.1$)$ & $<0.001$ & $-1.8(-2.6$ to -1.0$)$ & $<0.001$ & NR & NR & $-1.5(-2.2$ to -0.7$)$ & $<0.001$ & $-1.4(-2.2$ to -0.7$)$ & $<0.001$ \\
\hline Age 36 & HTT & NR & NR & 70 (-80 to 220$)$ & 0.356 & 59 (-89 to 207$)$ & 0.430 & 70 (-73 to 214$)$ & 0.338 & 70 (-74 to 241$)$ & 0.339 \\
\hline Age 43 & HTT & NR & NR & $-35(-131$ to 62$)$ & 0.443 & $-36(-133$ to 61$)$ & 0.464 & $-27(-131$ to 77$)$ & 0.613 & $-17(-127$ to 93$)$ & 0.761 \\
\hline Age 53 & HTT & NR & NR & 27 (-16 to 70$)$ & 0.221 & $25(-18$ to 69$)$ & 0.248 & 40 ( -3 to 83$)$ & 0.067 & $42(-2$ to 85$)$ & 0.059 \\
\hline Age 60-64 & HTT & NR & NR & $-25(-58$ to 9$)$ & 0.149 & NR & NR & $1.4(-33$ to 35$)$ & 0.936 & $2.9(-32$ to 37$)$ & 0.871 \\
\hline
\end{tabular}

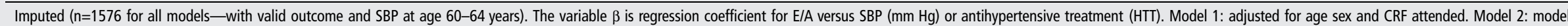

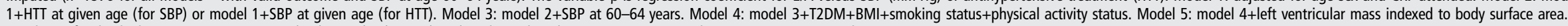
Numbers of individuals receiving HTT were $51(2 \%), 107(3 \%), 438(15 \%)$ and $640(27 \%)$ at age $36,43,53$ and $60-64$ years, respectively.

BMI, body mass index; CRF, clinical research facility; NR, not relevant; SBP, systolic blood pressure; T2DM, type 2 diabetes mellitus. 


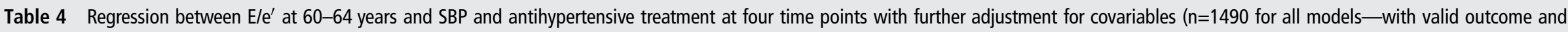
SBP at age 60-64 years)

\begin{tabular}{|c|c|c|c|c|c|c|c|c|c|c|c|}
\hline \multirow[b]{2}{*}{ Independent variable } & & \multicolumn{2}{|l|}{ Model 1} & \multicolumn{2}{|l|}{ Model 2} & \multicolumn{2}{|l|}{ Model 3} & \multicolumn{2}{|l|}{ Model 4} & \multicolumn{2}{|l|}{ Model 5} \\
\hline & & $\boldsymbol{\beta}(95 \% \mathrm{Cl})$ & $p$ Value & $\boldsymbol{\beta}(95 \% \mathrm{Cl})$ & $p$ Value & $\boldsymbol{\beta}(95 \% \mathrm{Cl})$ & $p$ Value & $\boldsymbol{\beta}(95 \% \mathrm{Cl})$ & $p$ Value & $\boldsymbol{\beta}(95 \% \mathrm{Cl})$ & p Value \\
\hline Age $36(n=1490)$ & SBP & 0.015 (0.006 to 0.024$)$ & 0.001 & 0.015 (0.006 to 0.024$)$ & 0.001 & $0.007(-0.002$ to 0.016$)$ & 0.118 & $0.008-(0.001$ to 0.017$)$ & 0.072 & $0.006(-0.003$ to 0.015$)$ & 0.170 \\
\hline Age $43(n=1490)$ & SBP & 0.019 (0.011 to 0.027$)$ & $<0.001$ & $0.017(0.009$ to 0.025$)$ & $<0.001$ & $0.008(0.000$ to 0.017$)$ & 0.052 & 0.009 (0.001 to 0.017$)$ & 0.031 & $0.009(0.000$ to 0.017$)$ & 0.045 \\
\hline Age $53(n=1490)$ & SBP & 0.022 (0.017 to 0.028$)$ & $<0.001$ & $0.020(0.014$ to 0.026$)$ & $<0.001$ & 0.013 (0.007 to 0.019$)$ & $<0.001$ & 0.011 (0.003 to 0.017 ) & 0.001 & $0.010(0.003$ to 0.016$)$ & 0.003 \\
\hline Age 60-64 $(n=1490)$ & SBP & $0.024(0.019$ to 0.030$)$ & $<0.001$ & 0.023 (0.018 to 0.029$)$ & $<0.001$ & NR & NR & 0.021 (0.015 to 0.027$)$ & $<0.001$ & $0.020(0.014$ to 0.025$)$ & $<0.001$ \\
\hline Age 36 & HTT & NR & NR & $-0.06(-1.18$ to 1.06$)$ & 0.917 & $0.06(-1.05$ to 1.18$)$ & 0.909 & $0.004(-1.08$ to 1.09$)$ & 0.994 & $0.01(-1.06$ to 1.08$)$ & 0.981 \\
\hline Age 43 & $\mathrm{HTT}$ & NR & NR & 1.38 (0.62 to 2.13 ) & $<0.001$ & 1.34 (0.59 to 2.08$)$ & $<0.001$ & $1.13(0.39$ to 1.87$)$ & 0.003 & $1.03(0.30$ to 1.75$)$ & 0.006 \\
\hline Age 53 & $\mathrm{HTT}$ & NR & NR & 0.72 (0.39 to 1.05 ) & $<0.001$ & 0.72 (0.39 to 1.04 ) & $<0.001$ & $0.62(0.30$ to 0.95$)$ & $<0.001$ & 0.55 (0.23 to 0.88 ) & 0.001 \\
\hline Age $60-64$ & HTT & NR & NR & $0.69(0.44$ to 0.95$)$ & $<0.001$ & NR & NR & 0.55 (0.29 to 0.82$)$ & $<0.001$ & $0.48(0.21$ to 0.75$)$ & $<0.001$ \\
\hline
\end{tabular}

The variable $\beta$ is regression coefficient for E/e' versus SBP ( $\mathrm{mm} \mathrm{Hg}$ ) or antihypertensive treatment (HTT). Model 1: adjusted for age sex and CRF attended. Model 2: model 1+HTT at given age (for SBP) or model 1+SBP at given age (for HTT). Model 3: model 2+SBP at 60-64 years. Model 4: model 3+T2DM+BMI+smoking status+physical activity status. Model 5: model 4+left ventricular mass indexed to body surface area. Numbers of individuals receiving HTT were 51 (2\%), $107(3 \%), 438$ (15\%) and $640(27 \%)$ at age $36,43,53$ and $60-64$ years, respectively.

BMI, body mass index; CRF, clinical research facility; NR, not relevant; SBP, systolic blood pressure; T2DM, type 2 diabetes mellitus.

Table 5 Regression between LAVI at 60-64 years and SBP and antihypertensive treatment at four time points with further adjustment for covariables ( $\mathrm{n}=1417$ for all models - with valid outcome and SBP at age 60-64 years)

\begin{tabular}{|c|c|c|c|c|c|c|c|c|c|c|c|}
\hline \multirow{2}{*}{ Independent variable } & & \multicolumn{2}{|l|}{ Model 1} & \multicolumn{2}{|l|}{ Model 2} & \multicolumn{2}{|l|}{ Model 3} & \multicolumn{2}{|l|}{ Model 4} & \multicolumn{2}{|l|}{ Model 5} \\
\hline & & $\boldsymbol{\beta}(95 \% \mathrm{Cl})$ & p Value & $\boldsymbol{\beta}(95 \% \mathrm{Cl})$ & p Value & $\boldsymbol{\beta}(95 \% \mathrm{Cl})$ & p Value & $\boldsymbol{\beta}(95 \% \mathrm{Cl})$ & $\mathrm{p}$ Value & B $(95 \% \mathrm{Cl})$ & p Value \\
\hline Age $36(n=1417)$ & SBP & 0.042 (0.013 to 0.072$)$ & 0.005 & $0.041(0.012$ to 0.071$)$ & 0.006 & 0.035 (0.004 to 0.065$)$ & 0.025 & 0.38 (0.008 to 0.068$)$ & 0.014 & 0.024 ( -0.005 to 0.053$)$ & 0.103 \\
\hline Age $43(n=1417)$ & SBP & $0.042(0.013$ to 0.070$)$ & 0.004 & 0.040 (0.011 to 0.069$)$ & 0.006 & 0.021 (0.001 to 0.061$)$ & 0.042 & 0.035 (0.005 to 0.064$)$ & 0.021 & $0.026(-0.003$ to 0.055$)$ & 0.079 \\
\hline Age $53(n=1417)$ & SBP & $0.056(0.036$ to 0.075$)$ & $<0.001$ & 0.047 (0.027 to 0.068$)$ & $<0.001$ & $0.043(0.021$ to 0.065$)$ & $<0.001$ & 0.038 (0.016 to 0.060$)$ & 0.001 & 0.023 (0.023 to 0.045$)$ & 0.035 \\
\hline Age 60-64 ( $n=1417)$ & SBP & 0.030 (0.009 to 0.051$)$ & 0.004 & 0.028 (0.008 to 0.050$)$ & 0.007 & NR & $N R$ & $0.020(-0.013$ to 0.041$)$ & 0.066 & $-0.001(-0.023$ to 0.022$)$ & 0.944 \\
\hline Age 36 & HTT & NR & NR & $2.560(-1.650$ to 6.771$)$ & 0.232 & $2.769(-1.433$ to 6.971$)$ & 0.195 & $1.931(-2.262$ to 6.124$)$ & 0.365 & $2.022(-1.935$ to 5.979$)$ & 0.315 \\
\hline Age 43 & HTT & NR & NR & $2.179(-0.393$ to 4.751$)$ & 0.097 & $2.205(-0.352$ to 4.762$)$ & 0.091 & $1.388(-1.204$ to 3.981$)$ & 0.293 & $0.657(-1.826$ to 3.141$)$ & 0.603 \\
\hline Age 53 & HTT & NR & NR & 2.304 (1.139 to 3.469$)$ & $<0.001$ & 2.326 (1.161 to 3.491$)$ & $<0.001$ & 1.979 (0.799 to 3.159$)$ & 0.001 & $1.418(0.260$ to 2.576$)$ & 0.016 \\
\hline Age $60-64$ & HTT & NR & NR & 1.290 (0.667 to 2.512$)$ & 0.001 & - & NR & 1.084 (0.134 to 2.033$)$ & 0.025 & $0.6453(-0.466$ to 1.371$)$ & 0.334 \\
\hline
\end{tabular}

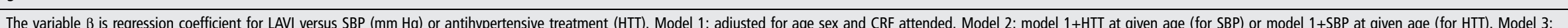

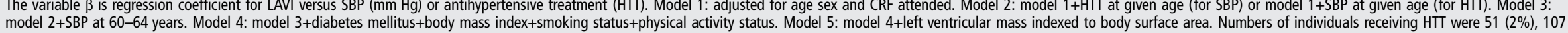
$(3 \%), 438(15 \%)$ and $640(27 \%)$ at ag $36,43,53$ and $60-64$ years, respectively.

CRF, clinical research facility; LAVI, left atrial volume indexed to body surface area; NR, not relevant; SBP, systolic blood pressure. 

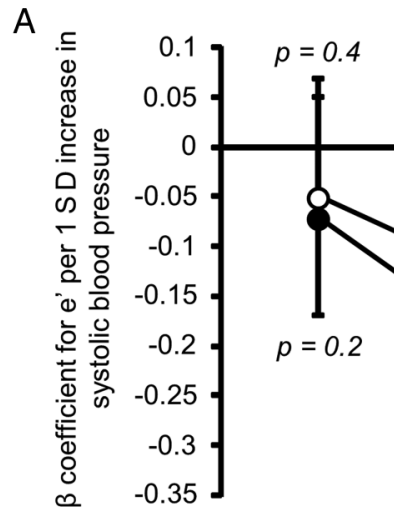

36-43 years

Period of rate of change in systolic blood pressure

$p<0.001$
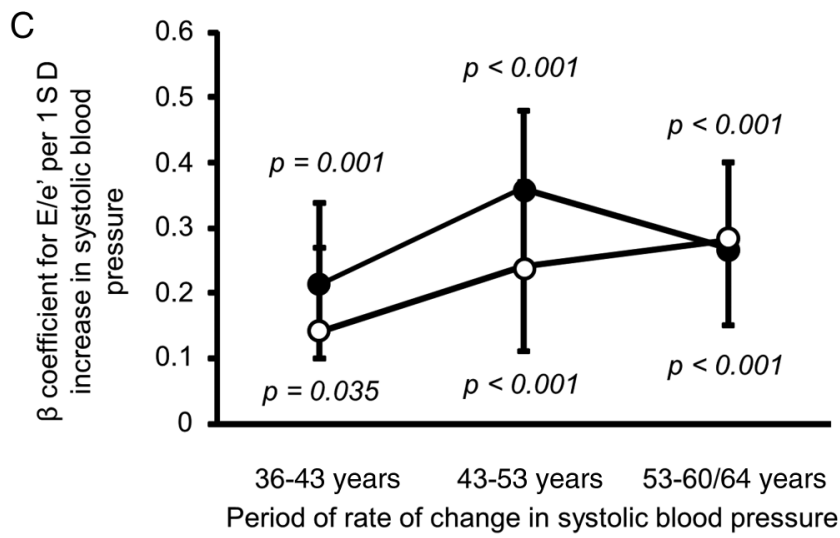

$\mathrm{B}$.

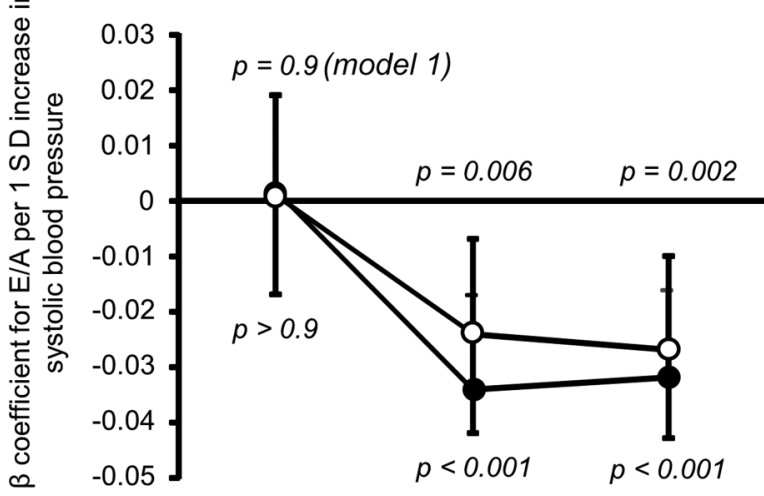

36-43 years $\quad 43-53$ years $53-60 / 64$ years

Period of rate of change in systolic blood pressure

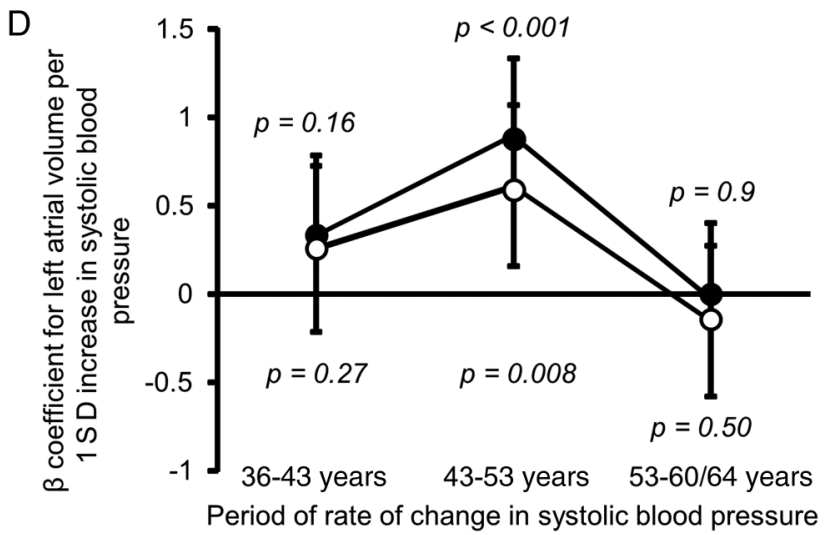

Figure 1 Association between standardised residuals of change in systolic blood pressure (SBP) over three time periods (36-43 years),

(43-53 years) and (53-60 to 64 years) conditional on the earlier measure(s) and markers of diastolic function at age 60-64 years. The residuals can be interpreted as the standardised rate of change in SBP in an individual above or below that expected on average in the sample given their earlier SBP. (A) $e^{\prime}$ at age 60-64 years $(n=1172)$. (B) E/A at age 60-64 years $(n=1190)$. (C) E/e' at age 60-64 years $(n=1120)$. (D) Left atrial volume indexed to body surface area at age $60-64$ years $(n=1054)$. Data points are $\beta$ coefficients and the bars represent $95 \%$ Cls. Model 1 ( $\bullet$ ): adjusted for age, sex and clinical research facility attended. Model $2(0)$ : model 1+diabetes mellitus+body mass index+smoking status+physical activity status+current antihypertensive treatment. $p$ Values were calculated using Wald tests and $p<0.05$ indicates a significant association between the rate of change in $\mathrm{SBP}$ in the specified period and the measure of diastolic function.

with decreased E/A measured 20 years later in the Swedish Uppsala cohort. ${ }^{16}$ Hypertension is thought to cause diastolic dysfunction via impaired LV relaxation and reduced LV compliance. ${ }^{17}$ Ultimately, the elevated filling pressures associated with severe diastolic dysfunction can lead to $\mathrm{HFPEF}^{18}$ or pulmonary oedema. ${ }^{19}$ Interestingly, Lee et al reported that higher BP and BMI in midlife were predictive of incident heart failure in later life in the Framingham study. ${ }^{4}$ Our data suggest that this relationship may be mediated at least in part via diastolic dysfunction.

Randomised clinical trials such as Antihypertensive and Lipid Lowering Treatment to Prevent Heart Attack Trial (ALLHAT) and HYpertension in the Very Elderly Trial (HYVET) have demonstrated the benefits of treating hypertension in terms of decreased incidence of new heart failure and heart failure admissions. ${ }^{20}$ However, optimal management of diastolic dysfunction remains unclear: trials in HFPEF have demonstrated little or no benefit with conventional heart failure medication. ${ }^{21}$ There is evidence for improvement in diastolic function with short-term HTT, ${ }^{22}{ }^{23}$ but longer-term HTT was not associated with improvement of diastolic function despite regression of LV mass in a substudy of the Anglo Scandinavian Cardiac Outcomes trial. ${ }^{24}$ Nevertheless, BP control is considered a cornerstone of management of these patients. ${ }^{25}$ In our cohort we found that from 43 years onwards, for any given level of BP (including for BP in the normal range, those on HTT had worse diastolic function at 60-64 years than those not on HTT). These data should not be interpreted as indicating that antihypertensive therapy has a negative effect on diastolic function, the observational design of the study precludes any such interpretation. Possible explanations of this finding include: inadequate long-term control of BP despite treatment ${ }^{26} 27$-it should be noted that only approximately half of hypertensive participants were controlled to $<140 / 90 \mathrm{~mm} \mathrm{Hg}$ at age 60-64 years; or the accrual of some BP-related damage prior to initiation of treatment. If the latter were true, individuals at risk may need to be treated at an earlier age and/or to lower target BP.

Elevated antecedent BP is associated with LV hypertrophy, ${ }^{7}$ and this could contribute to impaired diastolic function; however, adjustment for LVMI only moderately attenuated associations between antecedent $\mathrm{BP}$ and diastolic function and it seems that associations, particularly for $\mathrm{E} / \mathrm{e}^{\prime}$ and LAVI, are independent of increased LVMI. Some cross-sectional studies have found PP to be the best predictor of diastolic function, ${ }^{28} 29$ but we found relatively similar associations between antecedent SBP, DBP, PP and MAP and diastolic function. 


\section{Strengths and limitations}

Our findings are derived from a nationally representative sample of native-born British people born in 1946. ${ }^{8}$ Using the life course approach in relating BP at different ages and change in BP over time to future diastolic function is unique and is a major strength of our study. This was only possible due to detailed repeated measurements of risk factors and recording of HTT in our study, which is the longest running birth cohort in the UK. Studies of birth cohorts have a number of advantages, notably that accounting for the effect of age is much less problematic. We used tissue Doppler as our primary measure of diastolic function, this has the advantage that it can distinguish normal diastolic function from pseudonormalisation. ${ }^{30}$ Pseudonormalisation will tend to attenuate relationships between antecedent $\mathrm{BP}$ and diastolic function assessed by $\mathrm{E} / \mathrm{A}$, and probably accounts for the generally weaker relationships seen using this measure of diastolic function. Missing data are inevitable in studies as long-running as the MRC NSHD ( $>60$ years), although participant retention was good in comparison with other cohorts. ${ }^{11}$ Compared with those study members who attended clinic for clinical examination and echocardiography, those who only had clinical examinations at home visits had higher BMI and heart rates. However, participation of relatively more healthy individuals in the echocardiography study is, if anything, likely to have weakened relationships between $\mathrm{BP}$ and diastolic function. There were no measurements of $\mathrm{BP}$ prior to 36 years, and hence we cannot comment on the importance of BP in earlier life periods. We are limited to identifying a sensitive period from three intervals as the study has only four measures of BP. This lack of BP measurements in limits our ability to determine whether BP control was optimal between measurements. Echocardiography was only carried out in the last round of data collection; hence the possibility of diastolic dysfunction preceding the rise in BP cannot be excluded, although such a relationship seems unlikely.

\section{CONCLUSIONS}

$\mathrm{BP}$ from the age of 36 years predicts diastolic function in people aged 60-64 years independently of current BP (for $\mathrm{E} / \mathrm{e}^{\prime}$ and LAVI); faster increases in BP in midlife are particularly detrimental. People on HTT have more adverse diastolic function even when current BP is taken into account suggesting that early risk factor modification may be important to prevent the adverse effects of $\mathrm{BP}$ on diastolic function.

\section{Key messages}

\section{What is already known on this subject?}

- High blood pressure (BP) is associated with diastolic dysfunction in cross-sectional studies.

- Very little is known about the longitudinal effects of high BP and changes in BP over adult life on future diastolic function.

\section{What might this study add?}

- High blood pressure (BP) levels, and rises in BP in midadult life adversely affect diastolic function up to 28 years later.

- Hypertensive individuals have worse diastolic function even when their current BP was taken into account.

How might this impact on clinical practice?

- Early identification of those with fast rising blood pressure (BP) (even in the 'normal' BP range) may be important to prevent diastolic dysfunction in later life.
Acknowledgements We thank the NSHD study members who took part in the clinic data collection for their continuing support. We thank members of the NSHD scientific and data collection teams at the MRC Unit for Lifelong Health and Ageing at $U C L$ and the six Clinical Research Facilities.

Contributors AKG, RH and ADH conceived and designed the research and carried out the statistical analyses. DK secured funding for the study. All authors critically reviewed the manuscript. AKG, $\mathrm{ADH}$ and $\mathrm{RH}$ are responsible as guarantors for the overall content.

Funding The authors are/were employees of one of the following organisations: Barts Health NHS Trust, University College London or Imperial College. DF, ADH and JM received support from the Imperial College Academic Health Sciences Biomedical Research Centre. NC, JD and ADH received support from the University College Academic Health Sciences Biomedical Research Centre. NSHD, AKG, RH and DK were supported by the UK Medical Research Council (MC_UU_12018/1 and MC_UU_12019/2).

Competing interests None declared.

Ethics approval The Central Manchester Local Research Ethics Committee(07/H1008/168) and the Scotland A Research Ethics Committee.

Provenance and peer review Not commissioned; externally peer reviewed.

Open Access This is an Open Access article distributed in accordance with the terms of the Creative Commons Attribution (CC BY 4.0) license, which permits others to distribute, remix, adapt and build upon this work, for commercial use, provided the original work is properly cited. See: http://creativecommons.org/licenses/ by/4.0/

\section{REFERENCES}

1 Redfield MM, Jacobsen SJ, Burnett JC, et al. Burden of systolic and diastolic ventricular. Intern Med 2003;289:194-202.

2 Halley CM, Houghtaling PL, Khalil MK, et al. Mortality rate in patients with diastolic dysfunction and normal systolic function. Arch Intern Med 2011;171:1082-7.

3 Bella JN, Palmieri V, Roman MJ, et al. Mitral ratio of peak early to late diastolic filling velocity as a predictor of mortality in middle-aged and elderly adults: the Strong Heart Study. Circulation 2002;105:1928-33. http://www.ncbi.nlm.nih.gov/ pubmed/11997279 (accessed 18 Aug 2014).

4 Lee DS, Massaro JM, Wang TJ, et al. Antecedent blood pressure, body mass index, and the risk of incident heart failure in later life. Hypertens 2007;50:869-76.

5 Allen N, Berry JD, Ning H, et al. Impact of blood pressure and blood pressure change during middle age on the remaining lifetime risk for cardiovascular disease: the cardiovascular lifetime risk pooling project. Circulation 2012;125:37-44.

6 Arnlov J, Lind L, Zethelius B, et al. Several factors associated with the insulin resistance syndrome are predictors of left ventricular systolic dysfunction in a male population after 20 years of follow-up. Am Heart J 2001;142:720-4.

7 Ghosh AK, Hardy RJ, Francis DP, et al. Midlife blood pressure change and left ventricular mass and remodelling in older age in the 1946 British birth cohort study. Eur Hear J 2014;35:3287-95.

8 Stafford M, Black S, Shah I, et al. Using a birth cohort to study ageing: representativeness and response rates in The National Survey of Health and Development. Eur J Ageing 2013;10:145-57.

9 Gray L, Lee I-M, Sesso HD, et al. Blood pressure in early adulthood, hypertension in middle age, and future cardiovascular disease mortality: HAHS (Harvard Alumni Health Study). J Am Coll Cardiol 2011;58:2396-403.

10 Stang A, Moebus S, Möhlenkamp S, et al. Algorithms for converting random-zero to automated oscillometric blood pressure values, and vice versa. Am J Epidemiol 2006;164:85-94.

11 Kuh D, Pierce M, Adams J, et al. Cohort profile: updating the cohort profile for the MRC National Survey of Health and Development: a new clinic-based data collection for ageing research. Int J Epidemiol 2011;40:e1-9.

12 Nagueh SF, Appleton CP, Gillebert TC, et al. Recommendations for the evaluation of left ventricular diastolic function by echocardiography. J Am Soc Echocardiogr 2009;22:107-33

13 Fatema K, Bailey KR, Petty GW, et al. Increased left atrial volume index: potent biomarker for first-ever ischemic stroke. Mayo Clin Proc 2008;83:1107-15.

14 Keijzer-Veen MG, Euser AM, van et al. A regression model with unexplained residuals was preferred in the analysis of the fetal origins of adult diseases hypothesis. J Clin Epidemiol 2005;58:1320-4.

15 Wills AK, Lawlor DA, Matthews FE, et al. Life course trajectories of systolic blood pressure using longitudinal data from eight UK cohorts. PLoS Med 2011;8:e1000440.

16 Arnlöv J, Lind L, Sundström J, et al. Insulin resistance, dietary fat intake and blood pressure predict left ventricular diastolic function 20 years later. Nutr Metab Cardiovasc Dis 2005;15:242-9.

17 Vasan RS, Benjamin EJ. Diastolic heart failure-no time to relax. N Eng/ J Med 2001;344:56-9.

18 Zile MR, Baicu CF, Bonnema DD. Diastolic heart failure: definitions and terminology Prog Cardiovasc Dis 2005;47:307-13. 
19 Gandhi SK, Powers JC, Nomeir AM, et al. The pathogenesis of acute pulmonary edema associated with hypertension. N Engl J Med 2001;344:17-22.

20 Beckett NS, Peters R, Fletcher AE, et al. Treatment of hypertension in patients 80 years of age or older. N Engl J Med 2008;358:1887-98.

21 Ahmed A, Rich MW, Fleg JL, et al. Effects of digoxin on morbidity and mortality in diastolic heart failure: the ancillary digitalis investigation group trial. Circulation 2006;114:397-403.

22 Solomon SD, Janardhanan R, Verma A, et al. Effect of angiotensin receptor blockade and antihypertensive drugs on diastolic function in patients with hypertension and diastolic dysfunction: a randomised trial. Lancet 2007:369:2079-87.

23 Tapp RJ, Sharp A, Stanton AV, et al. Differential effects of antihypertensive treatment on left ventricular diastolic function: an ASCOT (Anglo-Scandinavian Cardiac Outcomes Trial) substudy. J Am Coll Cardiol 2010;55:1875-81.

24 Barron AJ, Hughes AD, Sharp A, et al. Long-term antihypertensive treatment fails to improve E/e' despite regression of left ventricular mass: an Anglo-Scandinavian cardiac outcomes trial substudy. Hypertension 2014;63:252-8.
25 Shah SJ, Gheorghiade M. Heart failure with preserved ejection fraction: treat now by treating comorbidities. JAMA 2008;300:431-3.

26 Lehmann MV, Zeymer U, Dechend R, et al. Ambulatory blood pressure monitoring: is it mandatory for blood pressure control in treated hypertensive patients?: prospective observational study. Int I Cardiol 2013;168:2255-63.

27 Hill MN, Miller NH, Degeest S, et al. Adherence and persistence with taking medication to control high blood pressure. I Am Soc Hypertens 2011;5:56-63.

28 Haider AW, Larson MG, Franklin SS, et al. Systolic blood pressure, diastolic blood pressure, and pulse pressure as predictors of risk for congestive heart failure in the Framingham Heart Study. Ann Intern Med 2003;138:10-16.

29 Franklin SS, Levy D. Aging, blood pressure, and heart failure: what are the connections? Hypertension 2011;58:760-2.

30 Quiñones MA. Assessment of diastolic function. Prog Cardiovasc Dis 2005;47:340-55. 